\title{
Search for Supernova Neutrino Bursts with the Large Volume Detector
}

\author{
G. Bruno \\ INFN Laboratori Nazionali del Gran Sasso, Italy \\ E-mail: gianmarco.bruno@lngs.infn.it \\ W. Fulgione \\ INFN Laboratori Nazionali del Gran Sasso, Italy \\ E-mail: fulgione@to.infn.it
}

\author{
A. Molinario \\ INFN Laboratori Nazionali del Gran Sasso, Italy \\ E-mail: andrea.molinariodlngs.infn.it \\ C. Vigorito* on behalf of the LVD Collaboration \\ Università \& INFN Torino, Italy \\ E-mail: vigorito@to.infn.it
}

\begin{abstract}
The Large Volume Detector (LVD) in the INFN Gran Sasso National Laboratory, Italy, is a 1 kton liquid scintillator neutrino observatory mainly designed to study low energy neutrinos from gravitational stellar collapses. LVD is sensitive to core-collapse supernovae via neutrino burst detection with $100 \%$ efficiency over the entire Galaxy. In this paper we summarize the results of the last run, lasting from 2014, January $1^{\text {st }}$ to 2015 , May $25^{\text {th }}$ for a total live time of 508.1 days. In the lack of a positive observation in this data set and including all previously published results since 1992, we establish the following upper limit on the rate of core collapse and failed supernova explosions out to distances of $25 \mathrm{kpc}: 0.11 \mathrm{yr}^{-1}$ at $90 \%$ c.l.
\end{abstract}

The 34th International Cosmic Ray Conference,

30 July- 6 August, 2015

The Hague, The Netherlands

* Speaker. 


\section{Introduction}

Gravitational stellar collapses (GSC) are astrophysical events of great interest. Because of the complexity of the problem, the modeling of the physical processes is still in evolution, but it is in general accepted that the role of neutrinos is critical to allow the supernova to form out of a collapse [1].

The confirmed detection of the neutrino signal from the SN 1987A, which was located in the Large Magellanic Cloud, marked the beginning of a new era in neutrino astrophysics (e.g. in [2, 3, 4]) and, in spite of some unresolved controversies [5], opened the way for a new method of investigation: The Neutrino Astronomy.

At the time next event will occur in our Galaxy the correlated neutrino emission will be detected by several different detectors. All the experiments aiming at the detection of neutrino bursts from core collapse supernovae (ccSN) have to face the extremely low frequency of this events. One such rare event is expected to happen in the Galaxy every 30-50 years [6]. This implies the ability to set up detectors which last several years with a very high duty cycle.

Because light can be partially or totally absorbed by dust in the Galactic plane while neutrinos are not (see the recent discussion by [7]), large long-term neutrino detectors are the most suited ones to observe the Galaxy and search for ccSN. Neutrino detectors are also sensitive to collapsing objects that fails to explode becoming black holes, the so-called failed supernovae. Those are expected to emit a neutrino signal even stronger[8], although shorter in time, than ccSN.

\section{The LVD detector}

The Large Volume Detector (LVD), operating since June 1992, is a 1 kton liquid scintillator detector whose main purpose is to monitor the Galaxy looking for neutrino bursts from GSC [9]. The detector is located underground at a depth of $1400 \mathrm{~m}$ under rock (3600 m w.e.), in the INFN Gran Sasso National Laboratory (Italy). The experiment consists of an array of 840 scintillator counters, $1.5 \mathrm{~m}^{3}$ each, viewed from the top by three photomultipliers (PMTs) and arranged in a modular geometry. This modularity allows LVD to achieve a very high duty cycle, that is essential in the search of unpredictable sporadic events. Failures involving one or more counters do not affect, in general, other counters. The detector can be serviced during data-taking by stopping only the part of the detector (down to each individual counter) that needs maintenance. The modularity of the detector results in a dynamic active mass $\mathrm{M}_{a c t}$. The experiment has been in operation since 1992, June $9^{\text {th }}$ after a short commissioning phase, its mass increasing from $300 \mathrm{t}$ to its final one, $1000 \mathrm{t}$, at time of building phase completion in January 2001. Duty cycle and active mass along the experiment life, up to 2015, May $25^{\text {th }}$ are shown in Fig. 1.

\subsection{Neutrino interaction channels in LVD}

Neutrinos can be detected in LVD through charged current (CC) and neutral current (NC) interactions on proton, Carbon nuclei and electrons of the liquid scintillator. The scintillator detector is supported by an iron structure, whose total mass is about $850 \mathrm{t}$. This can also act as a target for neutrinos and antineutrinos, as the product of interactions in iron can reach the scintillator and 


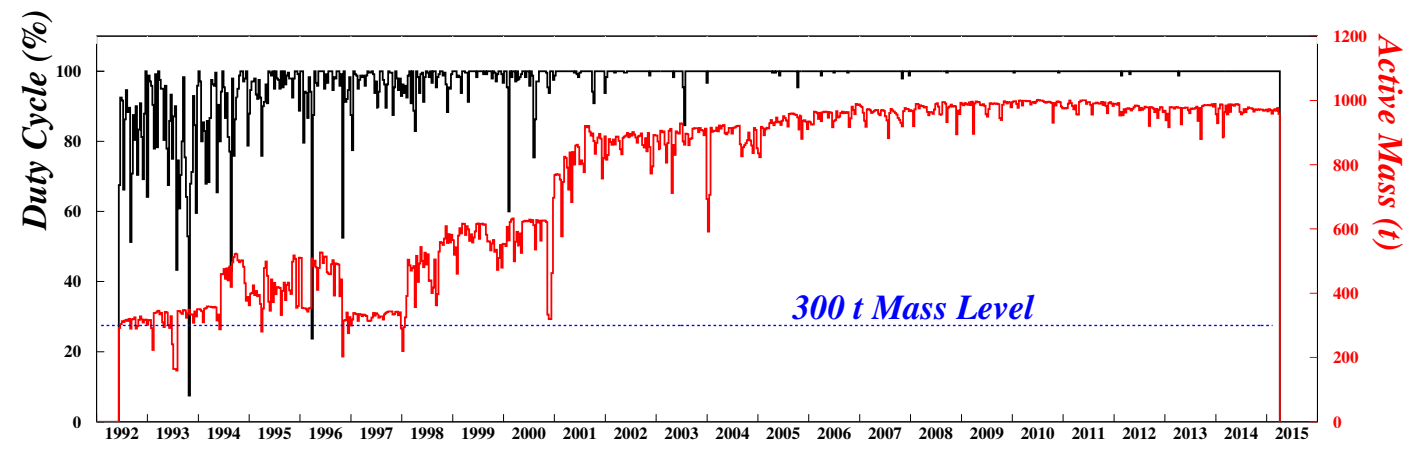

Figure 1: LVD duty cycle and active mass as a function of time from 1992, June $9^{\text {th }}$ to 2015, May $25^{\text {th }}$ : results on the data set since 2014 , January $1^{\text {st }}$ are discussed in the present update.

be detected [10]. The total target thus consists of $8.3 \cdot 10^{31}$ free protons, $4.3 \cdot 10^{31} \mathrm{C}$ nuclei and $3.4 \cdot 10^{32}$ electrons in the scintillator and of $9.7 \cdot 10^{30} \mathrm{Fe}$ nuclei in the support structure. The main neutrino reaction in LVD in case of a $v$ burst from GSC is the inverse beta decay (IBD), as it can be seen in Table 1, where all other relevant neutrino interaction channels are shown too. Given the relevance of the IBD, the LVD trigger has been optimized for the detection of both products of this interaction, namely the positron and the neutron. Each PMT is thus discriminated at two different threshold levels, the higher one $\left(\mathscr{E}_{H} \simeq 4 \mathrm{MeV}\right.$ in the present settings) is also the main trigger condition for the detector array. The lower one $\left(\mathscr{E}_{L} \simeq 0.5 \mathrm{MeV}\right)$ is in turn active only in a $1 \mathrm{~ms}$ time-window following the trigger, allowing the detection of $(\mathrm{n}, \mathrm{p})$ captures, the marker of a possible IBD interaction in the detector. Once a trigger is identified, the charge and time of the three summed PMTs signals are stored in a memory buffer. One millisecond after the trigger, all memory buffers are read out.

\begin{tabular}{cccc}
\hline \hline & $v$ interaction channel & $\mathrm{E}_{v}$ threshold & $\%$ \\
\hline 1 & $\bar{v}_{\mathrm{e}}+\mathrm{p} \rightarrow \mathrm{e}^{+}+\mathrm{n}$ & $(1.8 \mathrm{MeV})$ & $(88 \%)$ \\
2 & $v_{\mathrm{e}}+{ }^{12} \mathrm{C} \rightarrow{ }^{12} \mathrm{~N}+e^{-}$ & $(17.3 \mathrm{MeV})$ & $(1.5 \%)$ \\
3 & $\bar{v}_{\mathrm{e}}+{ }^{12} \mathrm{C} \rightarrow{ }^{12} \mathrm{~B}+e^{+}$ & $(14.4 \mathrm{MeV})$ & $(1.0 \%)$ \\
4 & $v_{\mathrm{i}}+{ }^{12} \mathrm{C} \rightarrow v_{\mathrm{i}}+{ }^{12} \mathrm{C}^{*}+\gamma$ & $(15.1 \mathrm{MeV})$ & $(2.0 \%)$ \\
5 & $v_{\mathrm{i}}+e^{-} \rightarrow v_{\mathrm{i}}+e^{-}$ & $(-)$ & $(3.0 \%)$ \\
6 & $v_{\mathrm{e}}+{ }^{56} \mathrm{Fe} \rightarrow{ }^{56} \mathrm{Co}^{*}+e^{-}$ & $(10 . \mathrm{MeV})$ & $(3.0 \%)$ \\
7 & $\bar{v}_{\mathrm{e}}+{ }^{56} \mathrm{Fe} \rightarrow{ }^{56} \mathrm{Mn}+e^{+}$ & $(12.5 \mathrm{MeV})$ & $(0.5 \%)$ \\
8 & $v_{\mathrm{i}}+{ }^{56} \mathrm{Fe} \rightarrow v_{\mathrm{i}}+{ }^{56} \mathrm{Fe}^{*}+\gamma$ & $(15 . \mathrm{MeV})$ & $(2.0 \%)$ \\
\hline
\end{tabular}

Table 1: The $v$ interaction channels detectable in LVD. The last column shows the percentages of events for any interaction channel in case of detection of a $v$ burst from GSC.

To evaluate the number of detected events in LVD for a neutrino burst from ccSN we consider a parameterized model, whose parameters have been determined by a maximum likelihood procedure on the data from SN 1987A [11]. From this model the average $\bar{v}_{e}$ energy is $\bar{E}_{\bar{v}_{e}}=14 \mathrm{MeV}$, the total 
radiated energy $E_{b}=2.4 \cdot 10^{53} \mathrm{erg}$. Energy equipartition and normal mass hierarchy for neutrino oscillations are assumed. If we set the distance of the collapsing star to $\mathrm{D}=10 \mathrm{kpc}$, we get that a total of 300 events (260 at $E_{v} \geq 10 \mathrm{MeV}$ ) are expected in LVD with a $1 \mathrm{kt}$ active mass, $88 \%$ of which are due to IBD (see details in [12]).

\section{Search for neutrino bursts}

LVD has been taking data since June 1992 with increasing mass configurations, being 300 $\mathrm{t}$ the minimal mass that allows LVD to be sensitive to neutrino bursts from GSC over the whole Galaxy, i.e. up to distance $D<25 \mathrm{kpc}$. The results of the search for neutrino bursts with LVD have been periodically reported at ICRCs (see [13] and references therein) and reviewed in a paper [14] covering the period 1992-2013. Here we focus on the results of the analysis of the last run, since 2014, January $1^{s t}$ to 2015 , May $25^{\text {th }}$, for a total live-time of 508 days and a global efficiency better than $99.8 \%$. The data set includes $5.5 \cdot 10^{8}$ triggers reduced to 1028250 in the [10-100] MeV energy range after muon rejection and quality cuts. The distribution of these triggers in time is shown in figure 2. Table 2 summarizes the features of the last run: average trigger rates before and after selections, active mass, total exposure and livetime.

\begin{tabular}{cccccccc}
\hline \hline & $R_{\text {tot }}$ & $R_{10}(E \geq 10 \mathrm{MeV})$ & $R_{L}(E \geq 0.5 \mathrm{MeV})$ & $\bar{M}_{\text {act }}$ & Exposure & live time & live time $(M \geq 300 \mathrm{t})$ \\
& {$\left[s^{-1} \cdot t^{-1}\right]$} & {$\left[s^{-1} \cdot t^{-1}\right]$} & {$\left[s^{-1} \cdot t^{-1}\right]$} & {$[\mathrm{t}]$} & {$[t \cdot$ year $]$} & {$[$ days $]$} & {$[$ days $]$} \\
\hline Last run & $135 \cdot 10^{-4}$ & $0.25 \cdot 10^{-4}$ & $2.4 \cdot 10^{2}$ & 936 & 1303 & 508 & 508 \\
\hline
\end{tabular}

Table 2: Data set features for the last run period.

After the selection cuts are applied the total counting rate $f$ results to be quite stable (bottom thick line in figure 2). The distribution of time intervals between consecutive triggers is shown in figure 3: differences have been normalized to a reference background rate $\left(\delta t_{\text {norm }}=\delta t \cdot f / f_{\text {ref }}\right.$, $f_{\text {ref }}=0.03 \mathrm{~s}^{-1}$ ) to take into account the variable detector configurations, as shown in figure 1 . The LVD events behave as a stochastic time series well described by the Poisson statistics, as shown by the quality of the fit to data in figure 3 .

\subsection{The selection algorithm}

To search for supernova neutrino bursts, we analize the time series of the selected events and look for clusters. While to provide the SNEWS, the on-line network of running neutrino detectors [15], with a prompt alert we use in the burst search method (on-line mode) a fixed time window (20s) [16], in this analysis (off-line mode) we consider different burst durations up to $100 \mathrm{~s}$ as discussed in detail in [17]. In both cases the selection is essentially a two-step process. Let us briefly review the latter here considered.

In the first step, we analyze the entire time series to search for clusters of events. The rationale of the search is that every n-th event could be the first of a possible neutrino burst. As we do not know a priori the duration of the burst, we consider all clusters formed by the $n$-th event and its successive ones. Namely, the $n$-th and the $(n+1)$-th ones define a cluster of multiplicity $m=2$; the $n$-th, $(n+1)$-th, $(n+2)$-th ones define another cluster of $m=3$, and so on and so forth. The duration 


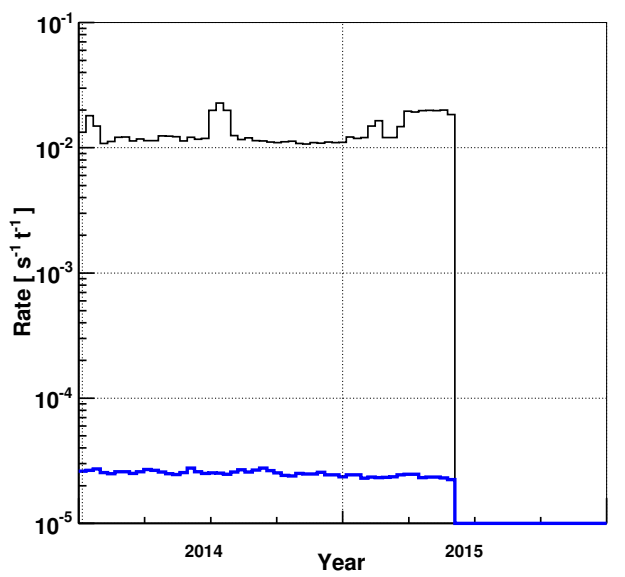

Figure 2: LVD counting rate as a function of time in the last run: top (thin) and bottom (thick) lines shows the total rate pre and post quality and energy selection cuts respectively.

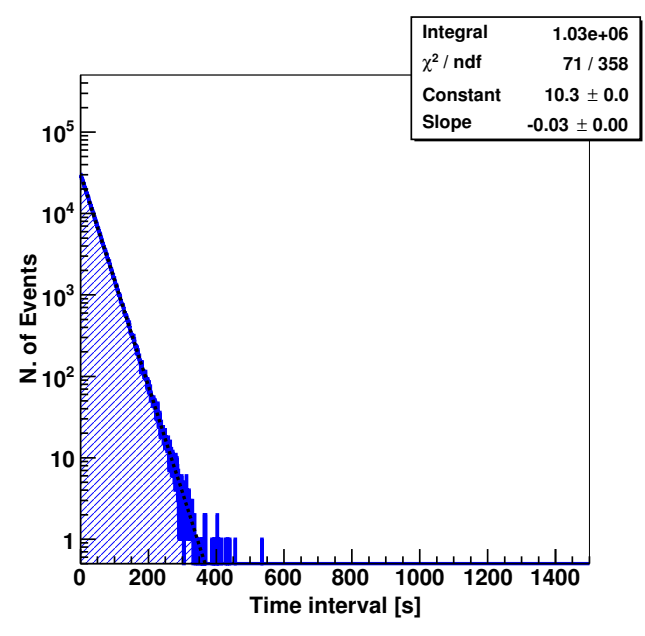

Figure 3: Distribution of normalized time intervals between triggers of the last run.

of each cluster is given by the time difference $\Delta$ t between the first event $n$-th and the last one of each sequence. When such a difference becomes larger than $\Delta \mathrm{t}_{\max }=100 \mathrm{~s}$, we do not consider that a cluster anymore: the analysis is then applied to the $(n+1)$-th event, and iteratively to all LVD events. The advantage of the described analysis, where all clusters with durations up to 100 $\mathrm{s}$ are considered, is that it is unbiassed with respect to the duration of the possible neutrino burst, unknown a priori. Moreover, the choice of $\Delta \mathrm{t}_{\max }=100 \mathrm{~s}$ is very conservative as it well exceeds the expected duration of a neutrino burst from ccSN and even more from failed supernovae.

The second step of the process consists in determining if one or more among the detected clusters are neutrino bursts candidates. To this aim, we associate to each of them (characterised by multiplicity $\mathrm{m}_{\mathrm{i}}$ and duration $\Delta t_{i}$ ) a quantity that we call imitation frequency $\mathrm{F}_{\mathrm{im}}$. This represents the frequency with which background fluctuations can produce, by chance, clusters with multiplicity $m \geq m_{i}$ and duration $\Delta t_{i}$. As shown in [17], this quantity, which depends on $\left(\mathrm{m}_{\mathrm{i}}, \Delta \mathrm{t}_{\mathrm{i}}\right)$, on the instantaneous background frequency, $\mathrm{f}_{\mathrm{bk}_{\mathrm{i}}}$ and on the maximum cluster duration chosen for the analysis, $\Delta \mathrm{t}_{\max }$, can be written as:

$$
\mathrm{F}_{\mathrm{im}}=\mathrm{f}_{\mathrm{bk}}^{2} \Delta \mathrm{t}_{\max } \sum_{\mathrm{k} \geq \mathrm{m}_{\mathrm{i}}-2} \mathrm{P}\left(\mathrm{k}, \mathrm{f}_{\mathrm{bk}_{\mathrm{i}}} \Delta \mathrm{t}_{\mathrm{i}}\right)
$$

where $\mathrm{P}\left(\mathrm{k}, \mathrm{f}_{\mathrm{bk}_{\mathrm{i}}} \Delta \mathrm{t}_{\mathrm{i}}\right)$ is the Poisson probability to have k events in the time window $\Delta \mathrm{t}_{\mathrm{i}}$ if $\mathrm{f}_{\mathrm{bk}}$ is the background frequency.

Given the duration of the LVD data set (more than 20 years), we choose $1 / 100 \mathrm{yr}^{-1}$ as imitation-frequency threshold, $F_{\mathrm{im}}^{\mathrm{th}}$. This means that a cluster $\left(\mathrm{m}_{\mathrm{i}}, \Delta \mathrm{t}_{\mathrm{i}}\right)$ is considered a neutrino burst candidate if:

$$
\sum_{\mathrm{k} \geq \mathrm{m}_{\mathrm{i}}-2} \mathrm{P}\left(\mathrm{k}, \mathrm{f}_{\mathrm{bk}_{\mathrm{i}}} \Delta \mathrm{t}_{\mathrm{i}}\right)<\frac{\mathrm{F}_{\mathrm{im}}^{\mathrm{th}}}{\mathrm{f}_{\mathrm{bk}}^{2} \cdot \Delta \mathrm{t}_{\max }} .
$$

The introduction of the imitation frequency has a double advantage. From the viewpoint of the search for neutrino bursts, it allows us to define a priori the statistical significance of each cluster 
in terms of frequency. Also, it allows us to monitor the performance of the search algorithm and the stability of the detector by increasing the imitation frequency threshold. Namely, we study the distributions of time differences between consecutive clusters for three different values of imitation frequency $\left(\mathrm{F}_{\mathrm{im}}<1\right.$ day $^{-1}$, week ${ }^{-1}$, month $\left.{ }^{-1}\right)$. Given the limited statistics we get when considering only the current run $\left(76,14\right.$ and 4 clusters detected with $\mathrm{F}_{\mathrm{im}}<1$ day $^{-1}$, week ${ }^{-1}$, month $^{-1}$ respectively), we perform this study including the data already published for the period 1992-2013 [14]. This results in a total of 1199, 179, 49 clusters for the 3 imitation frequencies, respectively. The distributions of the time differences between consecutive clusters are shown in figure 4 for $\mathrm{F}_{\mathrm{im}}<1$ day $^{-1}$ (black solid line), $\mathrm{F}_{\text {im }}<1$ week $^{-1}$ (green solid line) and $\mathrm{F}_{\mathrm{im}}<1$ month $^{-1}$ (blue solid line). The superimposed dotted lines are the result of a Poissonian fit to each distribution. The good agreement between data and the expected Poissonian behavior shows that the search algorithm and the detector are under control over the whole period of data taking.

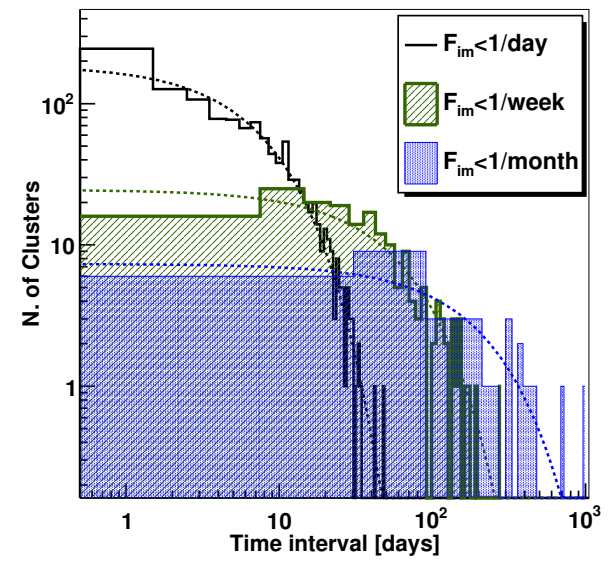

Figure 4: Distribution of time intervals beween consecutive clusters (solid lines) fitted by Poisson laws (dashed lines). $\quad$ All data since 1992 have been considered here.

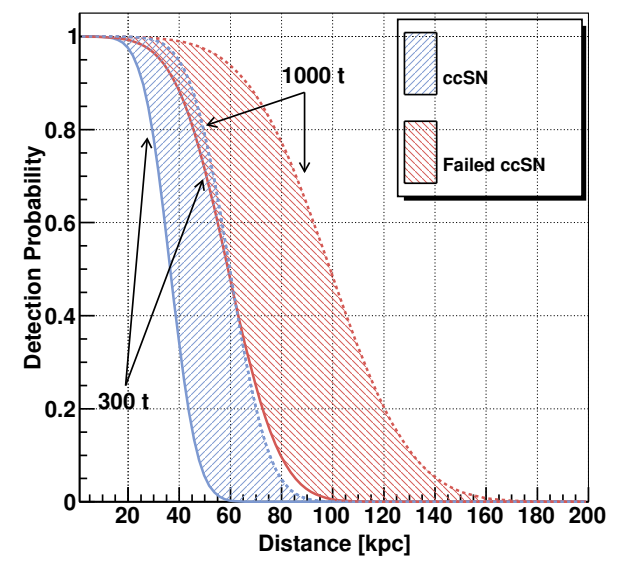

Figure 5: LVD detection probability versus source distance for the $F_{\text {im }}=1 / 100 \mathrm{yr}^{-1}$ for both ccSN and failed supernovae in the range of minimum (300 t) and maximum (1000 t) allowed active mass.

\section{LVD sensitivity}

The capabilities of LVD to detect a supernova event in both the on-line and off-line methods are extensively discussed in [16] and in [14] respectively. In the former a fixed time-window (i.e., $20 \mathrm{~s}$ ) is used while in the latter, which applies to the present analysis, we do not fix a priori the duration of the burst, i.e., we consider all possible durations up to $100 \mathrm{~s}$, as discussed in section 3.1. As shown in the two papers, the latter method is less model dependent than the former at a cost of a more complex procedure, which is not feasible on-line when the clusters selection has to be quite fast and reliable.

For the off-line method the detection probability as a function of the distance of the collapse is shown in Figure 5 for the chosen imitation frequency of $1 / 100 \mathrm{yr}^{1}$. The blue band corresponds to the case of standard ccSN: the solid (dashed) line represents an active mass of 300 (1000) t. 
The red band corresponds to the detection probability in the case of stellar collapses ending into black-holes (failed supernovae). In this case, we take as reference the predictions of Nakazato et al. (2008) [8] by choosing the most conservative one in terms of neutrino emission. According to that we can conclude that LVD is fully efficient to ccSN or failed supernovae within a radius of 25 $\mathrm{kpc}$ when the detector active mass is greater than $300 \mathrm{t}$.

\section{Results}

By analyzing the time series of 1028250 events of the last run, selected as described in Section 3 and collected over 508 days of data-taking, we get 2416878 clusters with multiplicity $m \geq 2$ and $\Delta \mathrm{t} \leq 100 \mathrm{~s}$.

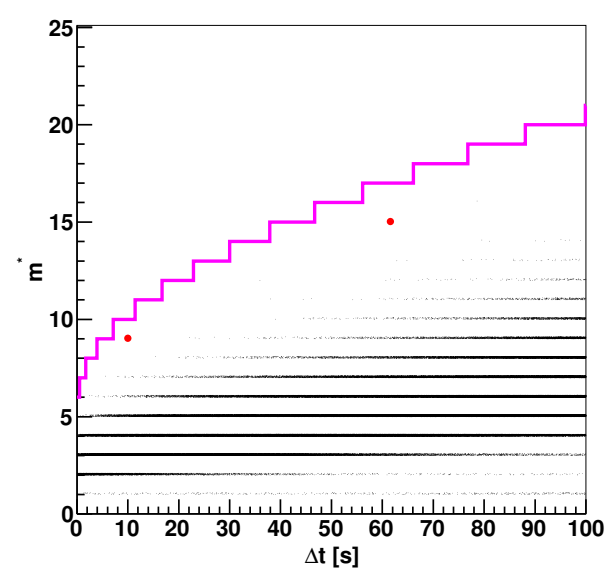

Figure 6: Distribution of detected clusters in the space $\left(\Delta t, m^{*}\right)$. Red dots represent clusters with imitation frequency less than $\mathrm{F}_{\mathrm{im}}=1 \mathrm{yr}^{-1}$. The purple line corresponds to $\mathrm{F}_{\mathrm{im}}^{\mathrm{th}}=0.01 \mathrm{yr}^{-1}$.

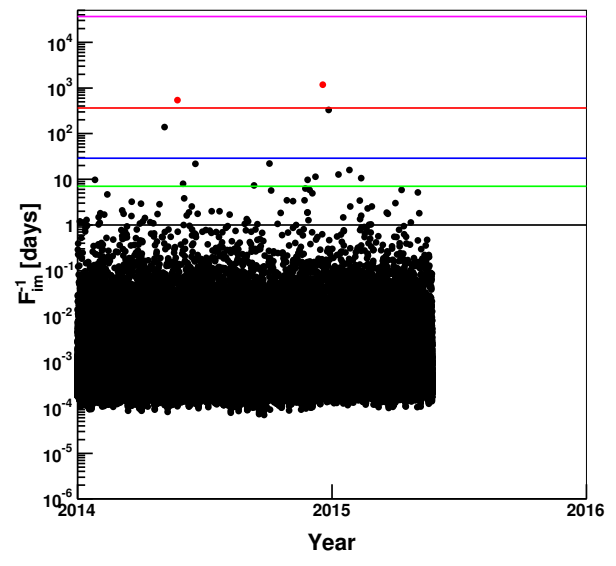

Figure 7: Inverse of the absolute imitation frequency of all detected clusters versus time for the present data set. Red dots represent clusters with imitation frequency less than $\mathrm{F}_{\mathrm{im}}=1 \mathrm{yr}^{-1}$. Black, green, blue, red and purple lines corresponds to $F_{\text {im }}^{\text {th }}=1$ day $^{-1}$, $\mathrm{F}_{\mathrm{im}}^{\text {th }}=1$ week $^{-1}, \quad \quad \mathrm{~F}_{\mathrm{im}}^{\mathrm{th}}=1 \mathrm{month}^{-1}$, $\mathrm{F}_{\mathrm{im}}^{\mathrm{th}}=1 \mathrm{yr}^{-1}, \quad \mathrm{~F}_{\mathrm{im}}^{\mathrm{th}}=0.01 \mathrm{yr}^{-1}, \quad$ respectively

Figure 6 shows in a two-dimensional graph the cluster duration, $\Delta \mathrm{t}$, and the normalized multiplicity, $m^{*}$, which takes into account the different instantaneous background rate at time of detection and allows to put all clusters toghether normalized to the reference background rate, $f_{\text {ref }}$. Details on the normalization procedure are discussed in [14]. Figure 7 shows the absolute imitation frequency of all clusters as a function of time. It is apparent that the occurrence of clusters with different $F_{\text {im }}$ over 508 days of measurement is quite uniform. In both figures 6 and 7 the purple line represents the expectations for a $F_{\text {im }}^{\text {th }}$ of $1 / 100 \mathrm{yr}^{-1}$, i.e., the threshold for considering a cluster as a neutrino-burst candidate. None of the observed clusters passes such threshold. Two clusters have a $F_{\text {im }}<1 / \mathrm{yr}$, being the maximum detected significance $\left(\mathrm{F}_{\mathrm{im}}\right)^{-1}=3.2 \mathrm{yr}$, associated to a cluster of 14 events lasting about $61 \mathrm{~s}$. Both clusters with $F_{i m}<1 / \mathrm{yr}$ have been checked in terms of energy 
spectra and low energy signals that may be the signature of the IBD interactions. They are fully compatible with chance coincidence among background signals. We conclude that no evidence is found for $\mathrm{ccSN}$ or failed supernovae during the considered data-taking period. Taking into account the total live-time of LVD, 7843 days, we obtain an upper limit on the rate of GSC out to $25 \mathrm{kpc}$ of 0.11 per year at $90 \%$ C.L..

\section{Conclusions}

In this paper we have presented the results of the search for neutrino-burst signals from supernovae explosions performed with LVD data taken over 508 days, since 2014, January $1^{\text {st }}$ to 2015 , May $25^{\text {th }}$. The neutrino-burst detection technique is based on the search for a sequence of candidate neutrino events whose probability of being simulated by fluctuations of the counting rate is very low. To select neutrino-bursts candidate among them we have searched for all possible clusters of events with durations up to $100 \mathrm{~s}$. The knowledge of the background as well as its long-term stability are of essence for evaluating the probability of each found cluster. Out of the 2.4 millions of detected clusters, we have found that none has an imitation frequency less than $1 / 100 \mathrm{yr}^{-1}$. We have thus concluded that no evidence has been found for GSC occurred up to $25 \mathrm{kpc}$ during the period of observation. Finally, taking into account all previous data since 1992 for a total livetime of 7843 days, we have set an upper limit of $0.11 \mathrm{yr}^{-1}$ at $90 \%$ C.L., this being the most stringent limit ever achieved by the observation of supernovae through neutrinos in the entire Galaxy.

\section{References}

[1] Bethe H.A. \& Wilson J.R. 1985, ApJ., 295, 14.

[2] Hirata, K et al. 1987, PhRvL, 58, 1490.

[3] Bionta R.M. et al. 1987, PhRvL, 58, 1494.

[4] Alekseev E.N. et al. 1987, JTPL, 45, 589.

[5] Aglietta M. et al. 1987, EL, 3, 1315.

[6] R.Diehl et al. 2006, Nature, 439, 45.

[7] Adams Scott M. et al., 2013, ApJ, 778:164.

[8] Nazakato k., Sumiyoshi K., Suzuki H. and Yamada S., 2008, PhRvD, 78, 083014.

[9] Aglietta, M. et al., 1992, NCimA, 105, 1793.

[10] Agafonova N.Yu. et al., 2007, APh, 27, 254-270.

[11] Pagliaroli G. et al. 2009, APh, 31, 163.

[12] Molinario A.2012, Ph.D. Tesys, Dep. of Physics, University of Torino.

[13] Fulgione W., Molinario A. and Vigorito C., 2013, Proc. of $33^{\text {rd }}$ ICRC (Rio de Janeiro).

[14] Agafonova N.Yu. et al., 2015, ApJ, 802, 47.

[15] Antonioli P. et al., 2004, NJPh, 6, 114.

[16] Agafonova N.Yu. et al., 2008, APh, 28, 516.

[17] Fulgione W., Mengotti-Silva N. \& Panaro L., 1996, NIMPA, 368, 512. 